\title{
Engineered Pathways for Correct Disulfide Bond Oxidation
}

\author{
Guoping Ren and James C.A. Bardwell
}

\begin{abstract}
Correct formation of disulfide bonds is critical for protein folding. We find that cells lacking protein disulfide isomerases (PDIs) can use alternative mechanisms for correct disulfide bond formation. By linking correct disulfide bond formation to antibiotic resistance, we selected mutants that catalyze correct disulfide formation in the absence of DsbC, Escherichia coli's PDI. Most of our mutants massively overproduce the disulfide oxidase DsbA and change its redox status. They enhance DsbA's ability to directly form the correct disulfides by increasing the level of mixed disulfides between DsbA and substrate proteins. One mutant operates via a different mechanism; it contains mutations in DsbB and CpxR that alter the redox environment of the periplasm and increases the level of the chaperone/protease DegP, allowing DsbA to gain disulfide isomerase ability in vivo. Thus, given the proper expression level, redox status, and chaperone assistance, the oxidase DsbA can readily function in vivo to catalyze the folding of proteins with complex disulfide bond connectivities. Our selection reveals versatile strategies for correct disulfide formation in vivo. Remarkably, our evolution of new pathways for correct disulfide bond formation in E. coli mimics eukaryotic PDI, a highly abundant partially reduced protein with chaperone activity. Antioxid. Redox Signal. 14, 2399-2412.
\end{abstract}

\section{Introduction}

D ISULFIDE BONDS ACT to stabilize many protein structures (8). The stability of disulfide-rich miniproteins, for instance, is almost entirely dependent upon disulfide bonds (9). Although disulfide bonds are important for folding and stability, their accurate formation is difficult to achieve. This is particularly true for proteins with high levels of disulfide connectivity, because the number of possible incorrect disulfides grows factorially as the number of cysteine residues increases. In the eukaryotic endoplasmic reticulum, protein disulfide isomerase (PDI), a thioredoxin fold protein, is thought to be a key player in establishing both the initial oxidation state of protein thiols and in establishing proper connectivity (20). PDI may be aided in these tasks by other thioredoxin-related proteins present in the endoplasmic reticulum and by the reduced glutathione (GSH)/ oxidized GSH (GSSG) redox buffer $(16,20)$. Thus, in eukaryotic organisms, the pathways responsible for the initial oxidation of disulfides and their proofreading or isomerization are thought to be substantially intermingled.

In contrast, in the bacteria Escherichia coli, it has long been thought that the disulfide oxidation and disulfide error correction systems are separate entities $(14,41)$. DsbA efficiently oxidizes substrates cotranslocationally by forming disulfides between cysteines that are adjacent in the amino acid se- quence (consecutive disulfides) $(6,25)$. DsbA is then reoxidized by the membrane bound protein $\operatorname{DsbB}(4,5)$. DsbA has very little proofreading or disulfide isomerase activity (43). Proteins with nonconsecutive disulfides are thought to require the action of the E. coli disulfide isomerase, DsbC, to correct mistaken disulfide linkages formed by DsbA (14). To attack incorrect disulfides, DsbC must be kept in a reduced form. This function is performed by the membrane protein DsbD, which in turn is kept reduced by the cytosolic thioredoxin, a thioredoxin-reductase and NADPH-dependent system $(26,28)$. DsbC may correct errors in disulfide formation via two principal mechanisms, a reductive mechanism and a direct isomerase mechanism $(18,37)$. Both mechanisms share a key step in which the reduced isomerase attacks incorrect disulfides, resulting in the formation of a mixed disulfide between the isomerase and the substrate protein. Although it has previously been suggested that DsbG, a protein related to DsbC, may assist in the isomerization of disulfide bonds as well, DsbG has almost no isomerization activity in vitro (22). Instead, it now appears that DsbG is involved in detoxifying sulfenic acid formation (13). Thus, DsbC appears to be the predominant disulfide isomerase present in E. coli, although it has been reported that DsbA has significant isomerase activity in vivo in that it is able to correctly express RNaseI a protein that contains one nonconsecutive disulfide bond (30). 
To generate a selection system that allows us to directly link correct disulfide bond formation to bacterial antibiotic resistance, we engineered an additional nonconsecutive disulfide into the ampicillin-resistance protein $\beta$-lactamase. This strategy opened up the possibility of selecting for $E$. coli variants that could catalyze disulfide bond isomerization in the absence of DsbC. The selected mutants were capable of efficiently expressing a variety of proteins with multiple disulfide containing proteins in an E. coli strain that lacks DsbC. All but one of these $d s b C$ bypass mutants act by massively overproducing DsbA, which apparently increases the level of mixed disulfides formed between DsbA and substrate proteins. Higher levels of mixed disulfides may in turn allow cells to directly perform correct oxidation of multidisulfide containing substrates. The remaining $d s b C$ bypass mutant that we identified contains mutations in both $d s b B$ and $c p x R$, which act to alter the periplasmic chaperone and redox environment and thus favor disulfide isomerization. These results show that by direct selection, we can easily direct the evolution of multiple alternative mechanisms for correct disulfide formation in vivo.

\section{Materials and Methods}

\section{Strains and growth conditions}

Strains and plasmids used in the study are listed in Table 1. Unless otherwise noted, cultures were grown at $37^{\circ} \mathrm{C}$ in LB medium supplemented with appropriate antibiotics. The knockout strains were generated through P1 transduction from the Keio collection stock. A dsbB1-148 truncation was generated by cam cassette insertion immediately after position G148 using the Wanner method (12) in RGP209. Then, the cam cassette was removed by $\mathrm{pCP} 20$ plasmid to obtain strain RGP1079. RGP1126 was generated by moving the cpxR56 allele from UVC56 to RGP1079 by P1 transduction using $k d g T:: k a n$ as a selection marker from the Keio collection of knockouts (3). Thus, RGP1126 is the reconstituted strain containing the two only essential mutations from UVC56. The kan cassette was then removed by pCP20 plasmid to obtain strain RGP1146.

\section{Plasmid construction and site-directed mutagenesis}

Point mutations were introduced into the wild-type bla gene in pBR322 using a Stratagene Quick Change kit (Stratagene) and appropriate mutagenic primers, and polymerase chain reaction was performed as directed. The product was digested with $D p n I$, precipitated using Pelletpaint ${ }^{\circledR}$ according to the protocol supplied by the manufacturer (Novagen)

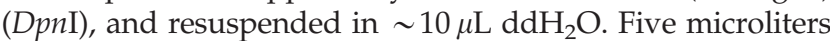
of product was transformed into TG1 competent cells (Stratagene). DNA was extracted from the cells and all point mutations were verified by DNA sequencing. Single disulfide bond $\beta$-lactamase mutants were made using a similar strategy but with multiple rounds of the procedure. Overexpression of DsbA was achieved by inserting the $d s b A$ gene into pBAD33 with restriction sites $\mathrm{XbaI}$ and HindIII. The MBP-bovine pancreatic trypsin inhibitor (BPTI) fusion protein was constructed by inserting a codon optimized BPTI after the MBP protein in the pMal-p2 (NEB) vector with the restriction sites SalI and HindIII. Then, the tobacco etch virus protease recognition site of ENLYFQS was immediately inserted before the BPTI sequence.

\section{Mutagenesis, screening, and mapping of mutants suppressing the $\mathrm{dsbC}^{-}$ampicillin phenotype}

RGP209 harboring pBR322 plasmid (R209 hereafter) was mutagenized with UV light. UV-irradiated cells were plated to nonselection (Tet) or selection (with $1.5 \mathrm{~g} / \mathrm{L}$ ampicillin) plates and grown overnight at $37^{\circ} \mathrm{C}$. Colonies appearing on the selection plates were screened on LB plates with $50 \mu \mathrm{M}$ cadmium to remove the loss-of-function mutants (broken disulfide bond formation machinery also suppresses the $d s b C^{-}$null strain phenotype as indicated in Fig. 1C). Colonies surviving the cadmium screen were restreaked to LB plates containing $1.5 \mathrm{~g} / \mathrm{L}$ ampicillin and subsequently to Tet plates. Suppressor candidates were then collected for further tests to rule out other falsepositive possibilities. Plasmids from the colonies were extracted and sequenced to guarantee no mutation in the bla gene. Polymerase chain reaction validation of the $d s b C^{-}$deletion in the genome was done to confirm no environmental bacterial contamination. Linkage of the mutations to the $d s b A$ loci was tested by $\mathrm{P} 1$ transduction using a yihG::Kan strain from the Keio collection. To move the $d s b A$ loci region of UVC49, the yigZ::Kan strain from the Keio collection was used as selection for P1 transduction. The $d s b A$ gene of each mutant was amplified with two primers $100 \mathrm{bp}$ upstream or downstream of $d s b A$ and subjected to sequence analysis. For suppressor strain UVC56, $d s b B$ and $d s b D$ genes were also amplified with primers $100 \mathrm{bp}$ upstream and downstream, respectively, and subjected to sequence analysis. To map the other mutations in the genome, we carried out genome sequencing using an Illumina ("Solexa") Genome Analyzer II. The genomic library was prepared according to Dr. Dave Lazinski, Tufts University. Of the 298 sequences tested, differences were observed between ER1821 and UVC56. Five candidate genes ( $y h j A, d s b B, d s b G$, pqiA, and $c p x R$ ) were further examined because they mapped to genes that link to envelope proteins potentially involved in protein folding. We checked these candidate genes by P1 transduction of both the wild-type genes and the knockout of these genes into UVC56.

\section{Spot titers for ampicillin resistance, cadmium resistance, and copper resistance}

Spot titers for ampicillin resistance were performed to test the $\beta$-lactamase or PDI detector $\beta$-lactamase activity in vivo. Cadmium resistance was performed to quantify the relative disulfide oxidase activity of the strains in vivo. Spot titers for copper resistance were performed to quantify the relative isomerase or reductase activity in vivo as described. Briefly, strains were grown overnight in LB and diluted 1:100 into fresh LB media with appropriate antibiotics. Strains were grown to mid-logarithmic phase at $37^{\circ} \mathrm{C}$ and serially diluted into $150 \mathrm{mM} \mathrm{NaCl}$. A $2 \mu \mathrm{L}$ aliquot of each dilution was plated onto LB plates with an ampicillin or cadmium gradient, or were plated onto terrific broth plates with $15-17 \mathrm{mM} \mathrm{CuCl}_{2}$. Cells were grown at $37^{\circ} \mathrm{C}$ overnight. All spot titers were performed at least in triplicate.

\section{Urokinase and BPTI activity assays}

Urokinase assays were performed on strains transformed with plasmid pRDB8-A, which expresses mouse urokinase plasminogen activator constitutively as described in Rietsch et al. (33). Periplasmic extract of bacteria grown to exponential phase was separated on nonreducing sodium dodecyl sulfate 
Table 1. Strains and Plasmids Used in This Study

\begin{tabular}{|c|c|c|}
\hline Strain name & Genotype/insert & Notes \\
\hline ER1821 (wild type) & $\mathrm{F}^{-} g \ln V 44$ e14- ${ }^{-}\left(\mathrm{McrA}^{-}\right)$rfbD1? relA1? endA1 spoT1? thi-1 $\Delta($ mcrC-mrr $) 114::$ IS10 & New England Biolabs \\
\hline RGP209 & ER1821 $d s b C^{-}$ & Lab stock \\
\hline RGP433 & ER1821 $d s b C^{-} d s b B^{-}$ & Lab stock \\
\hline RGP663 & ER1821 + pPDI detector & This study \\
\hline RGP664 & ER1821 $d s b A^{-}+$pPDI detector & This study \\
\hline RGP665 & RGP209+ pPDI detector (the starting strain) & This study \\
\hline RGP666 & ER1821 $d s b D^{-}+$pPDI detector & This study \\
\hline RGP667 & ER1821 $d s b A^{-} d s b C^{-}+$pPDI detector & This study \\
\hline RGP668 & ER1821 $\operatorname{tr} x A^{-}+$pPDI detector & This study \\
\hline RGP669 & ER1821 $\operatorname{tr} x B^{-}+$pPDI detector & This study \\
\hline RGP181 & ER1821 + pBR322 & This study \\
\hline RGP188 & ER1821 dsb $A^{-}+\mathrm{pBR} 322$ & This study \\
\hline RGP195 & ER1821 $d s b C^{-}+$pBR322 & This study \\
\hline RGP286 & ER1821 $d s b D^{-}+$pBR322 & This study \\
\hline RGP202 & ER1821 $d s b A^{-} d s b C^{-}+$pBR322 & This study \\
\hline RGP655 & ER1821 + pBla 81-108 & This study \\
\hline RGP685 & ER1821 + pBla 52-81 & This study \\
\hline RGP686 & ER1821 + pBla 98-108 & This study \\
\hline RGP719 & ER1821 + pBla 52-108 & This study \\
\hline RGP720 & ER1821 + pBla 81-98 & This study \\
\hline RGP829 $\rightarrow 834$ & UVC $49 \rightarrow 54, d s b C^{-}$suppressor strains & This study \\
\hline RGP843 $\rightarrow 848$ & UVC56 $\rightarrow 61, d s b C^{-}$suppressor strains & This study \\
\hline RGP852 & UVC62, $d s b C^{-}$suppressor strains & This study \\
\hline RGP877 $\rightarrow 880$ & UVC63 $\rightarrow 66, d s b C^{-}$suppressor strains & This study \\
\hline RGP849 & UVC49 cured the pBR322 plasmid & This study \\
\hline RGP851 & UVC52 cured the pBR322 plasmid & This study \\
\hline RGP1079 & ER1821 $d s b B 1-148 d s b C^{-}(d s b B$ was truncated as in UVC56) & This study \\
\hline RGP1126 & ER1821 dsbB1-148dsbC c cpxRM23I,E24K(kdgT::kan) & This study \\
\hline RGP1146 & RGP1126 kan cassette removed & This study \\
\hline RGP929 & RGP433 + pPDI detector & This study \\
\hline RGP982 & RGP1079+ pPDI detector & This study \\
\hline RGP916 & UVC56 $d s b A^{-}$ & This study \\
\hline RGP917 & UVC56 dsb $B^{-}$ & This study \\
\hline RGP918 & UVC56 $d s b D^{-}$ & This study \\
\hline RGP919 & UVC56 $d s b G^{-}$ & This study \\
\hline RGP920 & UVC56 umuD::kan $\left(d s b B^{+}\right)$ & This study \\
\hline RGP1073 & UVC56 kdgT::kan $\left(c p x R^{+}\right)$ & This study \\
\hline RGP1071 & UVC56 umuD ${ }^{-}\left(d s b B^{+}\right)$kdgT::kan $\left(c p x R^{+}\right)$ & This study \\
\hline RGP1181 & ER1821 + pRDB8-a & This study \\
\hline RGP1182 & RGP209+ pRDB8-a & This study \\
\hline RGP1183 & UVC49+ pRDB8-a & This study \\
\hline RGP1184 & UVC52+ pRDB8-a & This study \\
\hline RGP1185 & RGP1126+ pRDB8-a & This study \\
\hline RGP1244 & RGP209+ pRDB8-a + pBADDsbA & This study \\
\hline RGP1246 & RGP1079+ pBADDsbA & This study \\
\hline RGP860 & $\mathrm{ER} 1821+\mathrm{pM}$ PB & This study \\
\hline RGP862 & RGP209+ pMPB & This study \\
\hline RGP856 & UVC $49+$ pMPB & This study \\
\hline RGP858 & $\mathrm{UVC} 52+\mathrm{pMPB}$ & This study \\
\hline RGP1130 & RGP1126+ pMPB & This study \\
\hline RGP1256 & UVC56 $\operatorname{deg} P^{-}$ & This study \\
\hline RGP1269 & RGP665 yigZ::kan49 (dsbA loci from UVC49) & This study \\
\hline \multicolumn{3}{|l|}{ Plasmid name } \\
\hline pBR322 & With wild-type $\beta$-lactamase (Bla) in the vector & Lab stock \\
\hline pPDI detector & pBR322, Bla with mutations of S81C (TCA to TGT) and T108C (ACT to TGT) & This study \\
\hline pBla $81-108$ & pBR322 with Bla C52S C98S S81C T108C & This study \\
\hline pBla 52-81 & pBR322 with Bla C98S S81C & This study \\
\hline pBla 98-108 & pBR322 with Bla C52S T108C & This study \\
\hline pBla 81-98 & pBR322 with Bla C52S S81C & This study \\
\hline pRDB8-a & Urokinase constitutively active in the periplasm & Lab stock \\
\hline pMPB & MBP and BPTI fusion protein in the periplasm & This study \\
\hline pETDsbA & Codon optimized DsbA sequence & Lab stock \\
\hline pBADDsbA & DsbA expression induced by arabinose & This study \\
\hline
\end{tabular}

BPTI, bovine pancreatic trypsin inhibitor; MBP, maltose binding protein. 

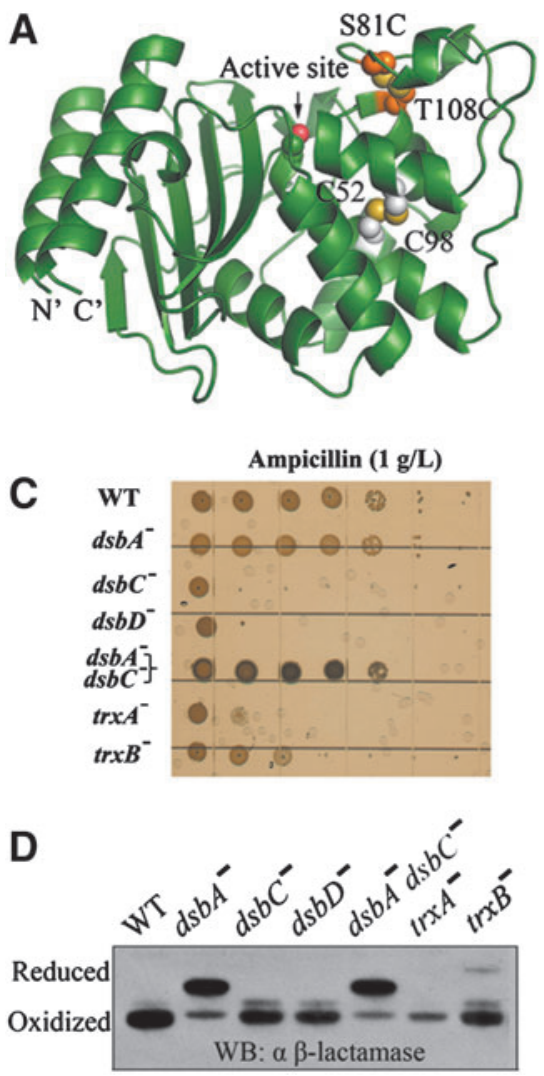

B
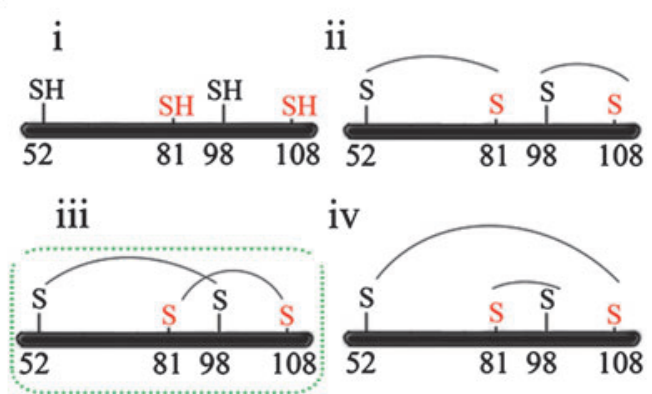

E

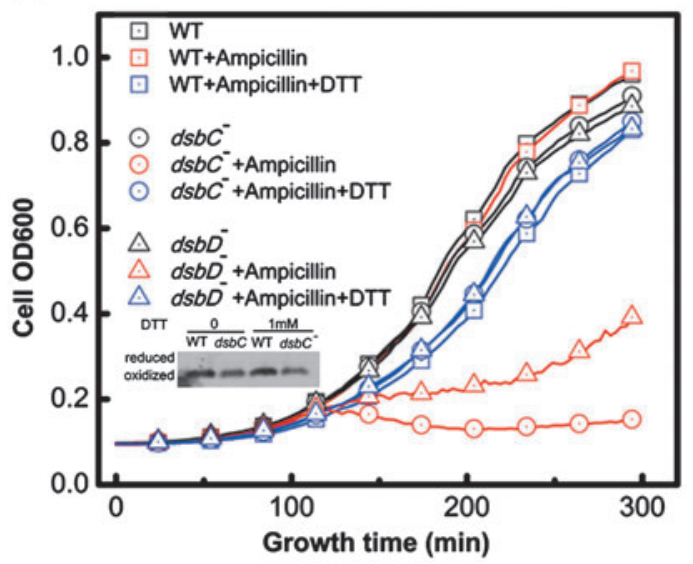

FIG. 1. Design and characteristics of a protein disulfide isomerase (PDI) detector $\beta$-lactamase construct that links correct disulfide bond formation to bacterial antibiotic resistance. (A) Model structure of the TEM1 $\beta$-lactamase PDI detector construct containing an engineered disulfide generated by cysteine substitutions at both serine 81 and threonine 108; model is based on the wild-type crystal structure $\quad(\mathrm{PDB}=1 \mathrm{BTL})$. (B) Possible patterns of disulfide bond linkage within the PDI detector $\beta$-lactamase. The native disulfide bond in $\beta$ lactamase is C52-C98. A native-like disulfide C81-C108 was engineered so as not to substantially disrupt the native structure. The native-like pattern (iii) is active (circled in green), whereas formation of other disulfides (ii and iv) inactivates $\beta$-lactamase (Fig. 2). Fully reduced PDI detector $\beta$-lactamase (i) is also expected to be active. (C) PDI detector $\beta$-lactamase exhibits decreased antibiotic resistance in Escherichia coli mutants that are deficient in disulfide isomerization. Mutants lacking any known component of the disulfide isomerization pathway, namely, thioredoxin reductase $\left(\operatorname{tr} x B^{-}\right)$, thioredoxin $\left(\operatorname{tr} x A^{-}\right), \operatorname{DsbD}\left(d s b D^{-}\right)$, or $\operatorname{DsbC}\left(d s b C^{-}\right)$, decrease the ampicillin resistance of strains containing the PDI detector. As expected from the full activity of reduced $\beta$-lactamase, strains containing the $d s b A-$ mutation including $d s b A^{-}$ and $d s b A^{-} d s b C^{-}$are ampicillin resistant. Spot titrations were performed on LB plates containing $1 \mathrm{~g} / \mathrm{L}$ ampicillin (Amp) for various strains transformed with the PDI detector construct in RGP663 (wild type), RGP664 (dsb $\left.A^{-}\right)$, RGP665 (dsbC $\mathrm{R}^{-}$), RGP666 $\left(d s b D^{-}\right)$, RGP667 ( $\left.d s b A^{-} d s b C^{-}\right)$, RGP668 $\left(\operatorname{tr} x A^{-}\right)$, and RGP668 (trx $\left.B^{-}\right)$. (D) Analysis of the in vivo oxidation status of PDI detector $\beta$-lactamase by 4 -acetamido- $4^{\prime}$-maleimidylstilbene-2,2'-disulfonic acid (AMS) trapping. The PDI detector is oxidized in the wild-type strain, reduced in $d s b A^{-}$and $d s b A^{-} d s b C^{-}$strains, and oxidized in $d s b C^{-}, d s b D^{-}$, trx $x A^{-}$, and $\operatorname{tr} x B^{-}$ strains. (E) Addition of $1 \mathrm{~m} M$ of the reductant DTT restores the growth of PDI detector $\beta^{-}$lactamase in $d s b C^{-}$and $d s b D^{-}$ strains on media containing $1 \mathrm{~g} / \mathrm{L}$ ampicillin. DTT, dithiothreitol. (To see this illustration in color the reader is referred to the web version of this article at www.liebertonline.com/ars).

FIG. 2. The C52-C98 or C81-C108 disulfides are the only ones that allow $\beta$-lactamase to retain activity. (A) In vivo activity of one disulfide bond containing $\beta$-lactamase mutants. The Western blot after acid-AMS-trapping at the far left shows the expression level and redox status (all oxidized) of each variant as measured from whole cells using $\beta$-lactamase antibody. The schemes in the middle indicate the names and linkages of the one disulfide bonded $\beta$-lactamase variants (engineered cysteines are shown in red; wild-type cysteines are shown in black). The spot titer panel at the right shows their ampicillin $(0.2 \mathrm{~g} / \mathrm{L})$ resistance. The single disulfide bonded variants showed decreased ampicillin resistance compared to wild-type $\beta$ lactamase. (B) A wild-type strain containing wild-type $\beta$-lactamase (52-98) (RGP181) grew well in liquid media containing $0.2 \mathrm{~g} / \mathrm{L}$ ampicillin in the presence or absence of DTT (upper graph), whereas $\beta$-lactamase (C81-C108) (RGP655) grew somewhat in $0.2 \mathrm{~g} / \mathrm{L}$ ampicillin, but only in the absence of DTT (lower graph). (C) $1 \mathrm{mM}$ DTT restores the ability of wild-type E. coli strains containing $\beta$-lactamase C52-C81 (RGP685) (upper graph) or $\beta$-lactamase C98-C108 (RGP686) (lower graph) to grow on media containing $0.2 \mathrm{~g} / \mathrm{L}$ ampicillin. (D) $1 \mathrm{mM}$ DTT also restores the ability of wild-type strains containing $\beta$-lactamase C52C108 (RGP719) (upper graph) or $\beta$-lactamase C81-C98 (RGP720) (lower graph) to grow in media containing $0.2 \mathrm{~g} / \mathrm{L}$ ampicillin. The requirement of disulfide bond catalysis machinery for $\beta$-lactamase activity. (E) Cell-titer curves for strains expressing PDI detector $\beta$-lactamase based on the spot titer assay on ampicillin gradient plates. Shown are wild type (RGP663), dsb $A^{-}$ (RGP664), $d s b C^{-}$(RGP665), $d s b D^{-}$(RGP666), $d s b A^{-} d s b C^{-}$(RGP667), and $\operatorname{tr} x A^{-}$(RGP668). (F) Cell-titer curves for strains expressing wild-type $\beta$-lactamase based on the spot titer assay on ampicillin gradient plates. Shown are wild type (RGP181), $d s b A^{-}$(RGP188), $d s b C^{-}$(RGP195), $d s b D^{-}$(RGP286), and $d s b A^{-} d s b C^{-}$(RGP202). (To see this illustration in color the reader is referred to the web version of this article at www.liebertonline.com/ars). 
(SDS)-polyacrylamide gel electrophoresis gels, and urokinase activity was demonstrated by assaying plasminogen activation by using plasminogen-casein agar. BPTI activity was analyzed by testing residual trypsin activity (according to the manufacturer Sigma-Aldrich instructions). A $50 \mu \mathrm{L}$ periplasmic extract was used for a trypsin inhibition assay in a $1 \mathrm{~mL}$ reaction using $\mathrm{N}-\alpha$-Benzoyl-L-arginine-4-nitranilide (Roche) as the substrate. About $0.5 \mu \mathrm{g}$ commercial BPTI was used as a positive control, and a periplasmic extract of cells expressing an empty vector was used as a negative control.

\section{Motility assay}

M9 media supplemented with $0.4 \%$ (v/v) glycerol as carbon source and free amino acids $(40 \mathrm{mg} / \mathrm{L}$ each) except cysteine was used to prepare $0.15 \%(\mathrm{w} / \mathrm{v})$ minimal media

A

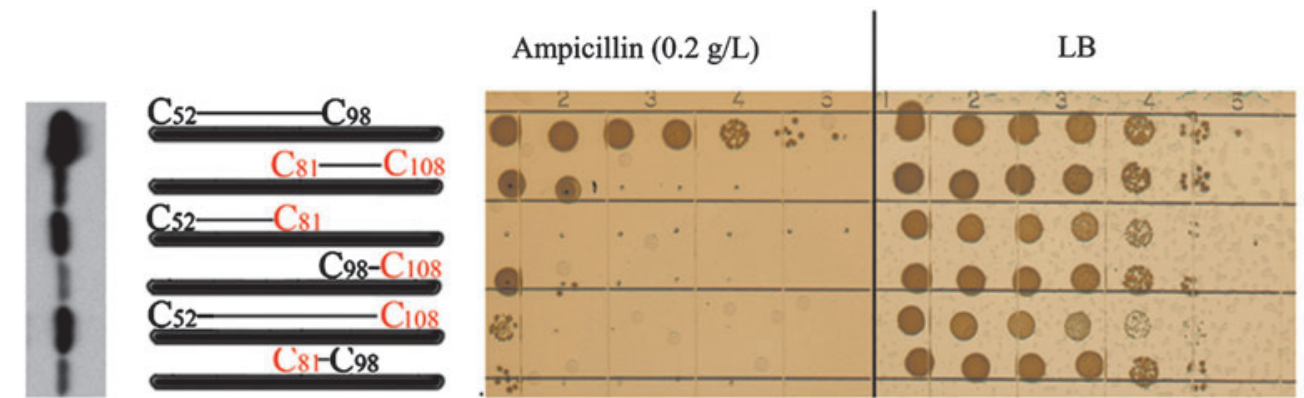

B
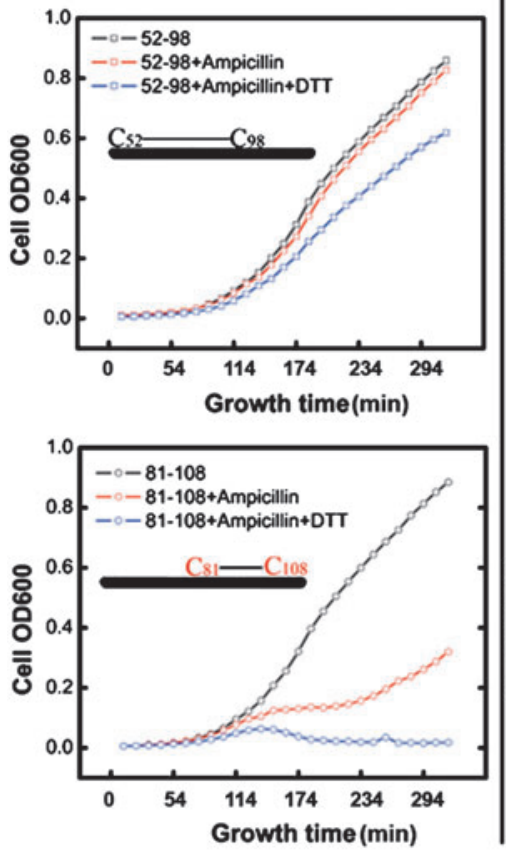

E

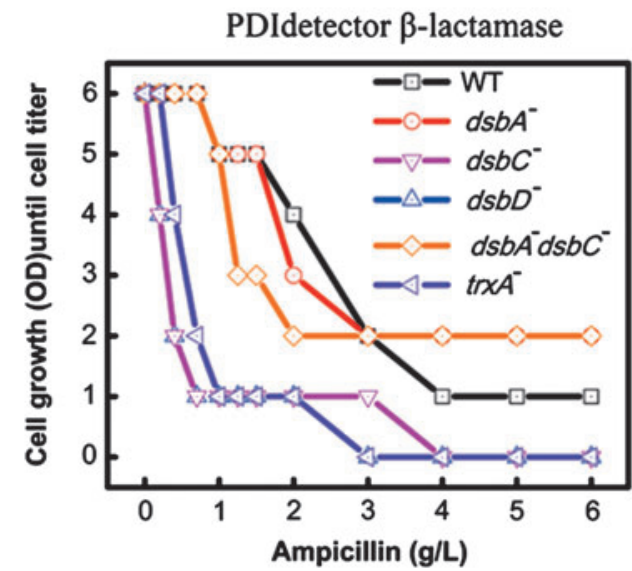

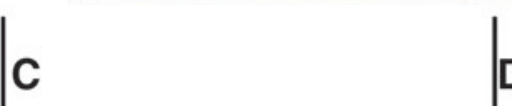
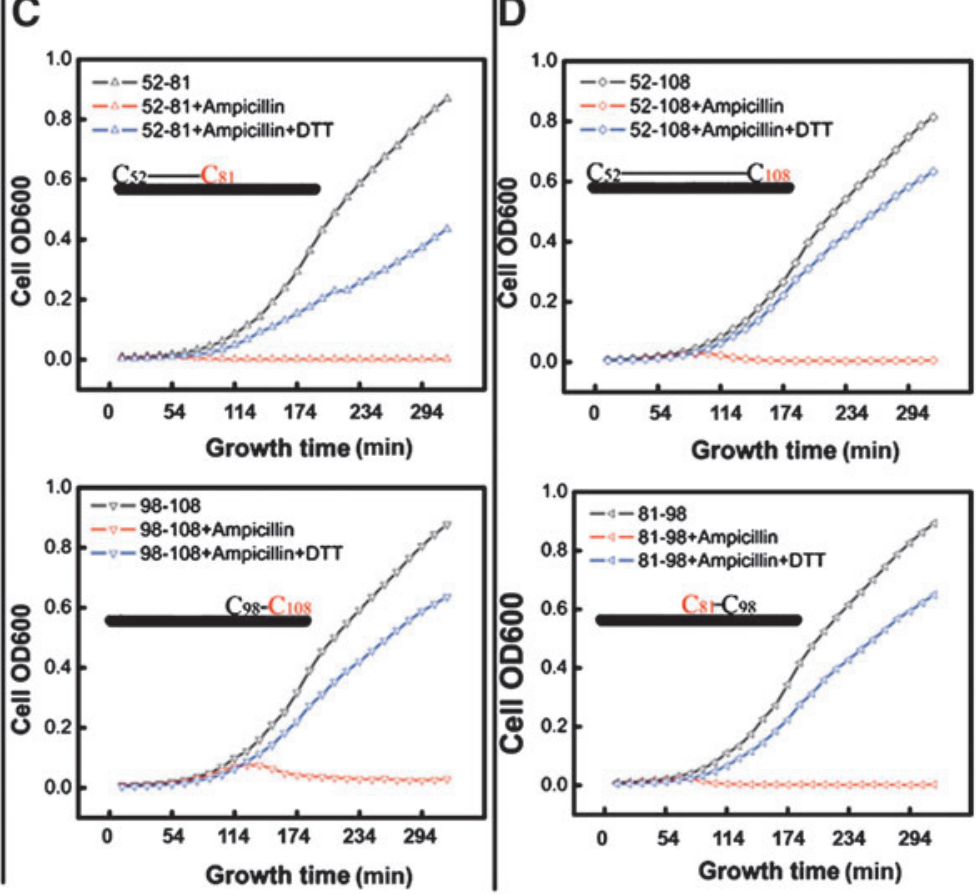

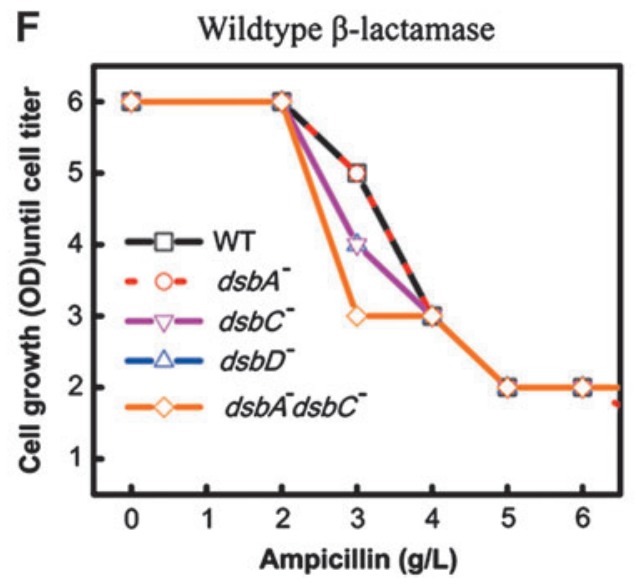


motility agar plates. Plates containing cystine and GSSG were also made by supplementing the above with $0.83 \mathrm{mM}$ cystine and $0.83 \mathrm{mM}$ GSSG, respectively. A single colony of the starting strain was inoculated in the middle of the plates, and inoculations of the various other strains were placed around the perimeter. After incubation at $37^{\circ} \mathrm{C}$ for $\sim 12 \mathrm{~h}$, the diameters of the various strains were observed.

\section{4-Acetoamido-4' -maleimidylstilbene-2,2' -disulfonic acid trapping}

Strains were grown to mid-logarithmic phase at $37^{\circ} \mathrm{C}$. Cells equivalent to 0.5 optical density units were acid-precipitated overnight. Acid-precipitated proteins were solubilized in buffered SDS solution containing $10 \mathrm{mg} / \mathrm{mL}$ 4-acetamido- 4 '-maleimidylstilbene-2,2'-disulfonic acid (AMS). The samples were incubated at $30^{\circ} \mathrm{C}$ for $30 \mathrm{~min}$, and $37^{\circ} \mathrm{C}$ for another $10 \mathrm{~min}$. Alkylation was stopped by the addition of reducing SDS loading buffer and analyzed by electrophoresis and Western blotting.

\section{Results}

\section{Linking the correct formation of disulfide bonds to bacterial antibiotic resistance}

To ascertain whether there are alternative mechanisms for disulfide isomerization, we decided to select for mutants that can bypass the requirement for the E. coli principal isomerase, DsbC. TEM1 $\beta$-lactamase is a periplasmic protein that has only a single and therefore consecutive disulfide bond (C52C98). Although this disulfide increases the folding stability of $\beta$-lactamase by $3.5 \mathrm{~kJ} / \mathrm{mol}$, it is not essential for ampicillin resistance (17). To make $\beta$-lactamase into a probe suitable for the detection of isomerase activity in vivo (a "PDI detector"), we designed an additional disulfide bond into the protein by substituting both serine 81 and threonine 108 with cysteine residues; these positions were chosen so that the connectivity of the engineered disulfide would span the native disulfide present in the protein's primary structure and, if formed correctly, would not substantially disrupt the protein tertiary structure (Fig. 1A, B). Serine 81 and threonine 108 were chosen because they are already very close together in the tertiary structure of $\beta$-lactamase. We reasoned that these mutations should convert $\beta$-lactamase into an active protein with two nonconsecutive disulfides when oxidized to its native-like conformation (Fig. 1B, iii). Because $\beta$-lactamase is secreted into the periplasm linearly (25), we would expect DsbA to initially oxidize the cysteines that are consecutive in the sequence (Fig. 1B, ii), resulting in an incorrect disulfide bonding pattern that very significantly distorts the structure, and thus decrease the activity and antibiotic resistance. Consistent with these considerations, we found that strains containing the $\beta$-lactamase PDI detector construct are only highly resistant to ampicillin when DsbC and other components of the DsbCDsbD disulfide isomerization pathway are present (Figs. 1C and $2 \mathrm{E}, \mathrm{F})$. In the absence of DsbC or DsbD, the $\beta$-lactamase PDI detector is almost fully oxidized (Fig. 1D) but its oxidation pattern appears to be incorrect, as these strains are sensitive to ampicillin. Addition of the small molecule dithiol DTT to the media restores the ampicillin resistance of strains containing the PDI detector construct (Fig. 1E). AMS trapping experiments shown in the insert to Figure $1 \mathrm{E}$ indicate that the PDI detector construct is fully oxidized in the presence of
DTT, implying that DTT is acting as a thiol disulfide exchange reagent that facilitates isomerization rather than a direct reductant. Our results suggest that improper oxidation of $\beta$ lactamase is inhibiting its ability to confer resistance. This was substantiated by the observation that in $d s b A^{-}$strains the PDI detector $\beta$-lactamase protein is almost fully reduced and the strains are ampicillin resistant (Fig. 1C), again consistent with the nonessential nature of the native disulfide in $\beta$-lactamase. Variants of the $\beta$-lactamase PDI detector construct with just a single pair of cysteines that are likely to form nonnative disulfides are sensitive to ampicillin but become resistant to ampicillin in the presence of DTT, providing further evidence that the formation of nonnative disulfides in $\beta$-lactamase interferes with its ability to encode ampicillin resistance (Fig. 2A-D). Our disulfide insertion design was based on the $\beta$-lactamase crystal structure that predicted that addition of the C81-C108 disulfide would not massively disrupt the $\beta$ lactamase structure. In contrast, all other disulfides, apart from the native C52-C98 bond, were expected to disrupt the structure and lead to large decreases in ampicillin resistance. The results shown in Figure 2 confirm these predictions: the wild-type protein containing the $\mathrm{C} 52-\mathrm{C} 98$ disulfide is the most ampicillin resistant, the variant containing the C81-C108 disulfide is somewhat resistant, and all others show lower levels of resistance. We note that the single disulfide bond variant of C81-C108 is not as active as either fully reduced or fully oxidized wild-type $\beta$-lactamase or fully oxidized PDI detector, as judged by ampicillin resistance. There are a number of possible explanations for this result: (i) The engineered disulfide C81-C108 may be suboptimal in geometry, resulting in a somewhat stained configuration that in the absence of the original native disulfide bond results in less enzymatic activity than either fully reduced or fully (correctly) oxidized $\beta$-lactamase. (ii) The cysteine52-serine and cysteine98-serine substitutions may not be entirely neutral, and may decrease the activity of the reduced form of variants containing this mutation. Evidence consistent with this possibility is the previous observation, made by Huang et al. (23) in their systematic study of active $\beta$-lactamase mutants, that cysteine52 and cysteine 98 could be only be substituted with only a very limited number of residues and still allow the $\beta$ lactamase protein to retain its ability to encode ampicillin resistance. Significantly, cysteine 98 could only be substituted with alanine, methionine, leucine, and isoleucine but not serine (23). Control experiments showed that the ampicillin resistance of wild-type $\beta$-lactamase is almost completely unaffected by DsbA, DsbC, or DsbD mutations (Fig. 2F), or by the presence or absence of DTT (Fig. 2B). Taken together, these data suggest that S81C T108C $\beta$-lactamase serves as a biomarker to monitor the disulfide isomerase activity present in a strain; henceforth, this construct is simply referred to as the PDI detector.

\section{$\mathrm{dsbC}^{-}$suppressors restore correct disulfide bond formation}

The ampicillin-sensitive phenotype of $d s b C^{-}$strains containing the PDI detector provides a powerful and convenient selection for host mutants that allow correct disulfide bond formation. We postulated that host mutations that bypass the need for the disulfide isomerase DsbC would allow strains containing the PDI detector to grow on ampicillin. Because 
reduced $\beta$-lactamase is active, strains that show strongly diminished rates of disulfide bond formation, due, for instance, to mutations in $d s b A$ or $d s b B$, should also result in ampicillin resistance. Strains containing the PDI detector construct were subjected to five independent rounds of UV mutagenesis, and colonies resistant to $1.5 \mathrm{~g} / \mathrm{L}$ ampicillin were selected as diagramed in Figure 3. Mutations in $d s b A$ or $d s b B$ result in sensitivity to the heavy metal cadmium (39). A large number of the mutants that we obtained were cadmium sensitive, indicating that they were either $d s b A^{-}$or $d s b B^{-}$; these mutants were not analyzed further. However, 17 mutants resistant to $1.5 \mathrm{~g} / \mathrm{L}$ ampicillin (4 are shown in Fig. 4A) were cadmium resistant and motile, indicating that they retained oxidase activity. These strains were named UVC49-54 and UVC56-66.

To test if these UVC mutants were enhancing ampicillin resistance through alterations in their ability to form disulfides and not via some disulfide irrelevant mechanism, such as by changing cell permeability to the antibiotic, we examined the periplasmic expression of two other multiple disulfide bond containing proteins whose folding had previously

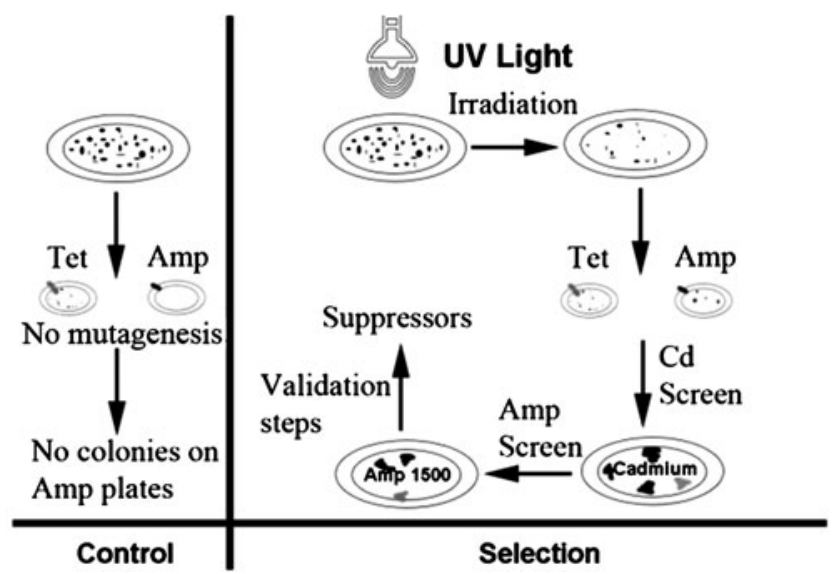

FIG. 3. Schematic illustrating the genetic selection of $d s b C^{-}$strain suppressors using the PDI detector. Ampicillin-resistant derivatives of the $d s b C^{-}$strain that contained the PDI detector $\beta$-lactamase plasmid were obtained following UV mutagenesis and plating on LB plates containing $1.5 \mathrm{~g} / \mathrm{L}$ ampicillin (right panel). No colonies exhibiting this level of ampicillin resistance could be obtained by directly plating the starting strain without mutagenisis (left panel). Ampicillin-resistant mutants obtained following mutagenesis (right panel) were then screened for cadmium sensitivity to eliminate mutants deficient in disulfide oxidation. Cadmium exerts its toxic effect by binding tightly to free thiols. Strains deficient in disulfide oxidation due to mutants in $d s b A$ or $d s b B$ have a high number of free thiols and are therefore cadmium sensitive (39) This provides an easy way to screen out $d s b A$ or $d s b B$ inactive mutants ( $\sim 95 \%$ of the ampicillinresistant colonies were eliminated in this secondary screening step). Mutants that were both ampicillin and cadmium resistant were streaked out for single colonies on plates containing $1.5 \mathrm{~g} / \mathrm{L}$ ampicillin. Plasmids from the candidate strains (named UVC49-UVC66) were sequenced to verify that no mutation had occurred within the PDI detector construct. Polymerase chain reaction was then used to verify that the strain still contained the original $d s b C$ deletion (UVC55 was excluded as it did contain the $d s b C$ gene). been shown to be DsbC dependent (urokinase and BPTI) (33) in three of the selected DsbC suppressors (UVC49, UVC52, and UVC56). We found that the activity of the multidisulfide proteins in these DsbC suppressors was restored to levels at or near those seen in $d s b C^{+}$strains (Figs. $4 \mathrm{~B}$ and $5 \mathrm{C}$ ). These results indicated that three different DsbC substrates are at least partially properly oxidized in these DsbC suppressor strains.

In screening these mutants for the presence of DsbA protein via Western analysis, we were struck by the fact that all the strains showed DsbA expression levels that were higher than those seen in wild-type backgrounds, with most of the DsbC suppressors exhibiting very high levels of expression (Fig. 4C). DsbA levels were $~ 50$-fold higher in most of the suppressors (UVC49-54, and UVC57-66, Fig. 5A); these were termed class I DsbC suppressors. UVC56 showed only $\sim 5$-fold higher DsbA levels and was termed a class II DsbC suppressor (Fig. 5A). Moving the wild-type DsbA locus into all 16 of the class I DsbC suppressors by $\mathrm{P} 1$ phage cotransduction resulted in restoration of the loss of ampicillin resistance (Table 2), showing that DsbC suppression activity for these mutants is genetically tightly linked to DsbA overexpression and is caused by a genetic alteration near the DsbA locus. In all cases the protein coding sequence of the DsbA gene was wild type. In contrast, introduction of the wild-type DsbA locus into UVC56, the single class II DsbC suppressor, did not decrease its ampicillin resistance, demonstrating that the DsbC suppressor mutation in this strain is unlinked to the DsbA locus (Table 2). In addition, DsbA overexpression off a plasmid in the class I DsbC suppressor UVC52 was sufficient to restore urokinase activity of a $d s b C$ null mutant to wild-type levels (Fig. 5D).

\section{The mechanism of class I dsbC ${ }^{-}$suppression by DsbA overproduction}

We examined the mechanism of action of the DsbC suppressors by conducting thiol trapping experiments. In these experiments, the thiol reactive reagent AMS was used to freeze thiol disulfide exchange (19). DsbA crossreactive material was then detected by Western blotting. This approach enabled us to determine the steady-state levels of oxidized and reduced DsbA present in vivo and to detect any mixed disulfides between DsbA and substrate proteins (Fig. 4D). Strikingly, the class I suppressors UVC49 and UVC52 show a massive accumulation (on a per cell basis) of high-molecularweight material that cross reacts with the DsbA antibody (Fig. $4 \mathrm{D}$, upper left panel). Because these bands are absent in the presence of DTT, they almost certainly represent disulfide linked DsbA-substrate complexes $(25,27)$. We also observed an increase in the percentage of reduced DsbA present (from $\sim 20 \%$ in wild-type strains to $\sim 50 \%$ in both the class I and II suppressors) (Fig. 4D, lower right panel). As we describe in the Discussion, the increased amounts of both reduced DsbA and disulfide-linked DsbA-substrate complexes in the class I suppressors may be important for the ability of these suppressors to catalyze correct disulfide bond formation.

\section{DsbC suppression in the class II suppressor, UVC56, requires mutations in both $\mathrm{dsbB}$ and $\mathrm{cpxR}$}

The second class of DsbC suppressor mutant is represented by a single example, UVC56. Unlike the class I suppressors, UVC56 does not accumulate mixed disulfides with substrates and is resistant to copper (Figs. 5E and 6D), implying that 
A

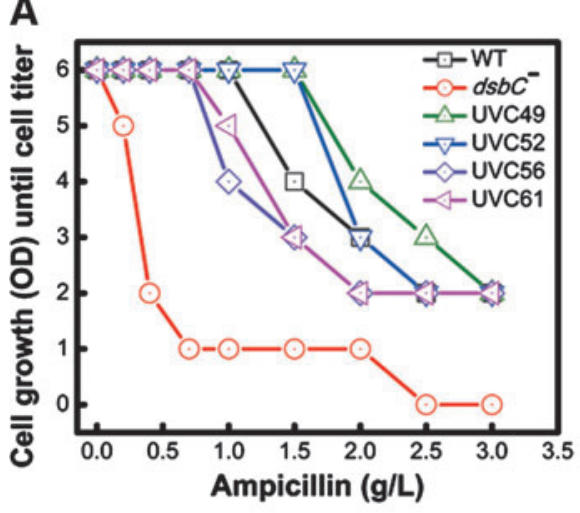

B

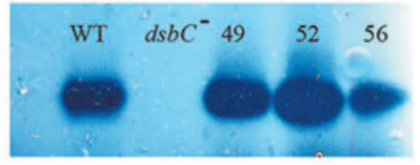

D

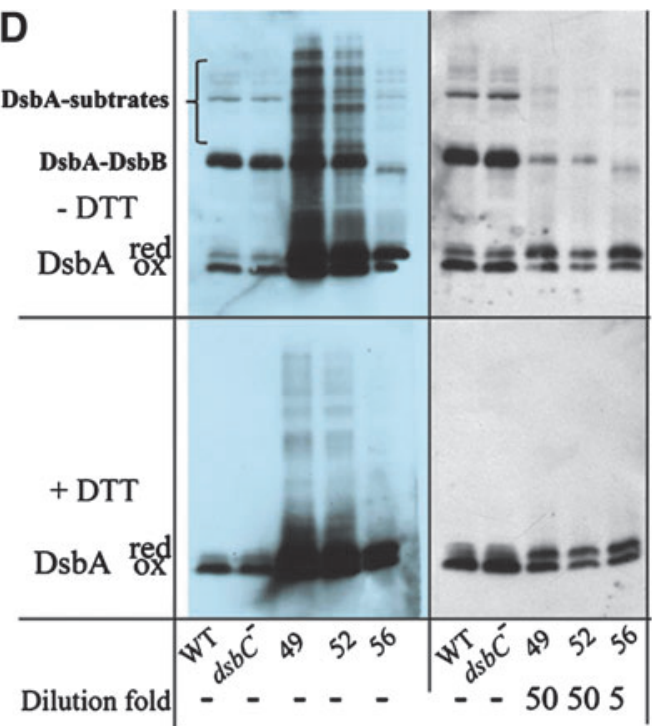

C

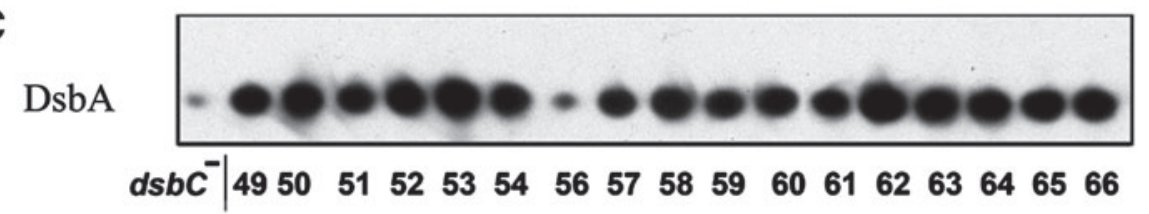

E

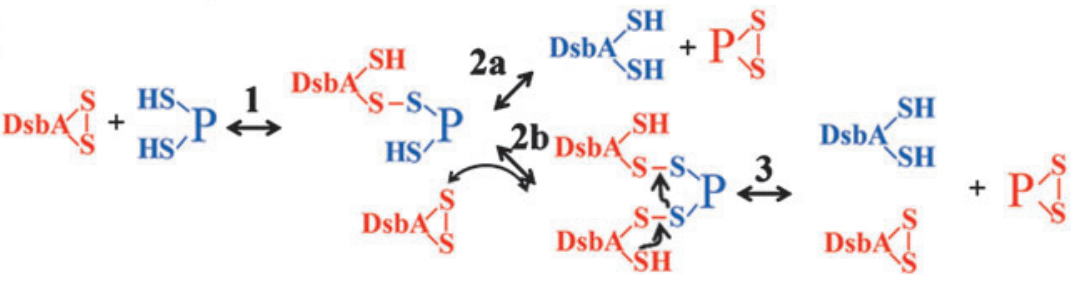

FIG. 4. Mutations that suppress the $d s b C^{-}$phenotype restore the ability to form disulfides correctly and greatly overexpress DsbA. (A) Restored ampicillin resistance of $d s b C^{-}$suppressor strains. Spot titrations were performed on LB plates containing various concentrations of ampicillin (Amp) using the following strains: wild type (RGP663), dsbC(RGP665), UVC49 (RGP829), UVC52 (RGP833), UVC56 (RGP843), and UVC61 (RGP848). (B) Restoration of mouse urokinase activity in three $d s b C^{-}$suppressor strains (UVC49, UVC52, and UVC56). Urokinase activity was detected by a casein agar assay as described in Materials and Methods section. Dark blue bands indicate activity. (C) DsbA is highly overexpressed in the $d s b C^{-}$suppressors. An equal quantity of bacterial cells of the various $d s b C$ suppressors was resolved on a reducing sodium dodecyl sulfate gel and the blot was probed with a DsbA antibody. The number under the bands indicates the suppressor's UVC number (e.g., 49 is UVC49), the $d s b C^{-}$

cells used in the first lane were from the starting, unmutagenized strain, RGP665. (D) Detection of DsbA and DsbA-substrate complexes in vivo in wild-type bacteria, $d s b C^{-}$strain, and in UVC49, UVC52, and UVC56 by Western analysis. Equal amounts of cells (left panels) were AMS acid trapped and loaded to nonreducing (upper panel) and reducing gels (lower panel). This allowed detection of the amount of DsbA present on a per cell basis. To get an idea of the relative amount of oxidized and reduced DsbA and DsbA-substrate complexes on a per DsbA basis, dilutions of each cell sample were made such that approximately equal quantities of DsbA protein were loaded (right panels). The UVC49 and UVC52 samples were thus diluted 50-fold and the UVC56 sample was diluted 5-fold before loading; all other samples were not diluted as indicated by a "-." The blots were probed with DsbA antibody. (E) Various pathways of substrate protein (abbreviated as P) oxidation by the DsbA protein. Disulfides are shown as S-S, free thiols as SH. Red indicates oxidized, blue reduced. (To see this illustration in color the reader is referred to the web version of this article at www.liebertonline.com/ars).

UVC56 is more proficient in disulfide isomerization than are the class I suppressors. To determine which mutations are present in the UVC56 strain, its genome was sequenced and compared with that of the starting strain. There were 298 differences found, but mutations in just two genes, a double mutation in cpxR56 (M23I E24K) and an IS10 insertion mutation in $d s b B$ at G135, accounted for nearly all of the suppression phenotypes. We showed this by constructing a strain (RGP1146) in which the cpxR56 and dsbB::IS10 mutations had been moved into the $d s b C$ null strain RGP209; we found that this strain very closely resembled UVC56 in its high levels of ampicillin, cadmium, and copper resistance (Figs. 6A, D and 7B). DsbB is the enzyme required for reoxidation of $\mathrm{DsbA}$, and CpxR is a positive regulator of the Cpx periplasmic stress response $(10,15)$. The Cpx regulon is a twocompontent signal transduction pathway that mediates adaptation to periplasmic protein misfolding and consists of a sensor histidine kinase ( $\mathrm{pxA})$ and a cyoplasmic response regulator (CpxR). DsbA is one of the genes under $c p x$ control (31). We found that reversion of the cpxR M23I E24K mutation decreased the level of DsbA present to wild-type levels, showing that the increase in DsbA expression observed for UVC56 was due to this cpxR mutation and implying that this mutation is constitutively active (Fig. 6B). Null mutants of $d s b B$ in which the entire $d s b B$ locus was replaced with a kanamycin resistance cassette gave the same level of suppression of ampicillin resistance of the PDI detector and the same cadmium resistance as the DsbB truncation, showing that this truncation is exerting its effect as a loss-of-function mutation. That a strain containing what was effectively a DsbB null mutation could oxidize proteins, as judged by its cadmium resistance, was initially surprising, given that DsbB reoxidizes DsbA and is thus normally very important for disulfide bond formation (5). However, the activity of DsbB can be replaced by adding small disulfide-containing molecules such as cystine and GSSG to the media (5). We found 
FIG. 5. DsbA overexpression is necessary and sufficient for the ability of the suppressor strains to express proteins with complex disulfide bond patterns. (A) DsbA is massively overexpressed in the suppressor strains as measured by Western blot $(\sim 50$-fold in most of the DsbC suppressors and $\sim 5$-fold in UCV56). Samples for suppressor strains were diluted 50 -fold before loading except for UVC56, which was diluted 5-fold. (B) Spot titer for strains expressing the PDI detector on LB plates and on those containing $0.6 \mathrm{~g} / \mathrm{L}$ ampicillin. The $d s b A$ gene from UVC49 was moved into the $d s b C^{-}$strain (RGP1269) by P1 cotransduction using the closely linked marker yigZ::kan. Strain genotypes are indicated at the far left: wild type (RGP663), dsb $C^{-}$(RGP665), UVC49 (RGP829), and $d s b C^{-}$ yigZ::kan (RGP1269). DsbA expression level as determined by Western blot of whole cell extracts using an-

A
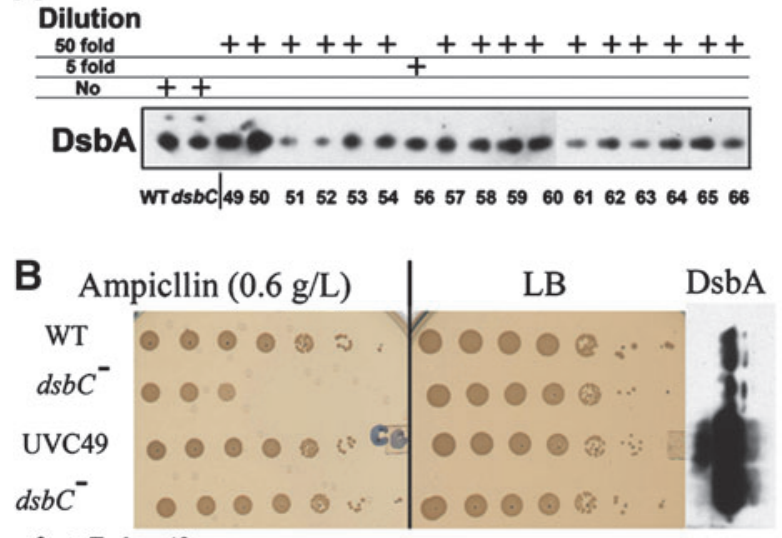

\&yigZ::kan49
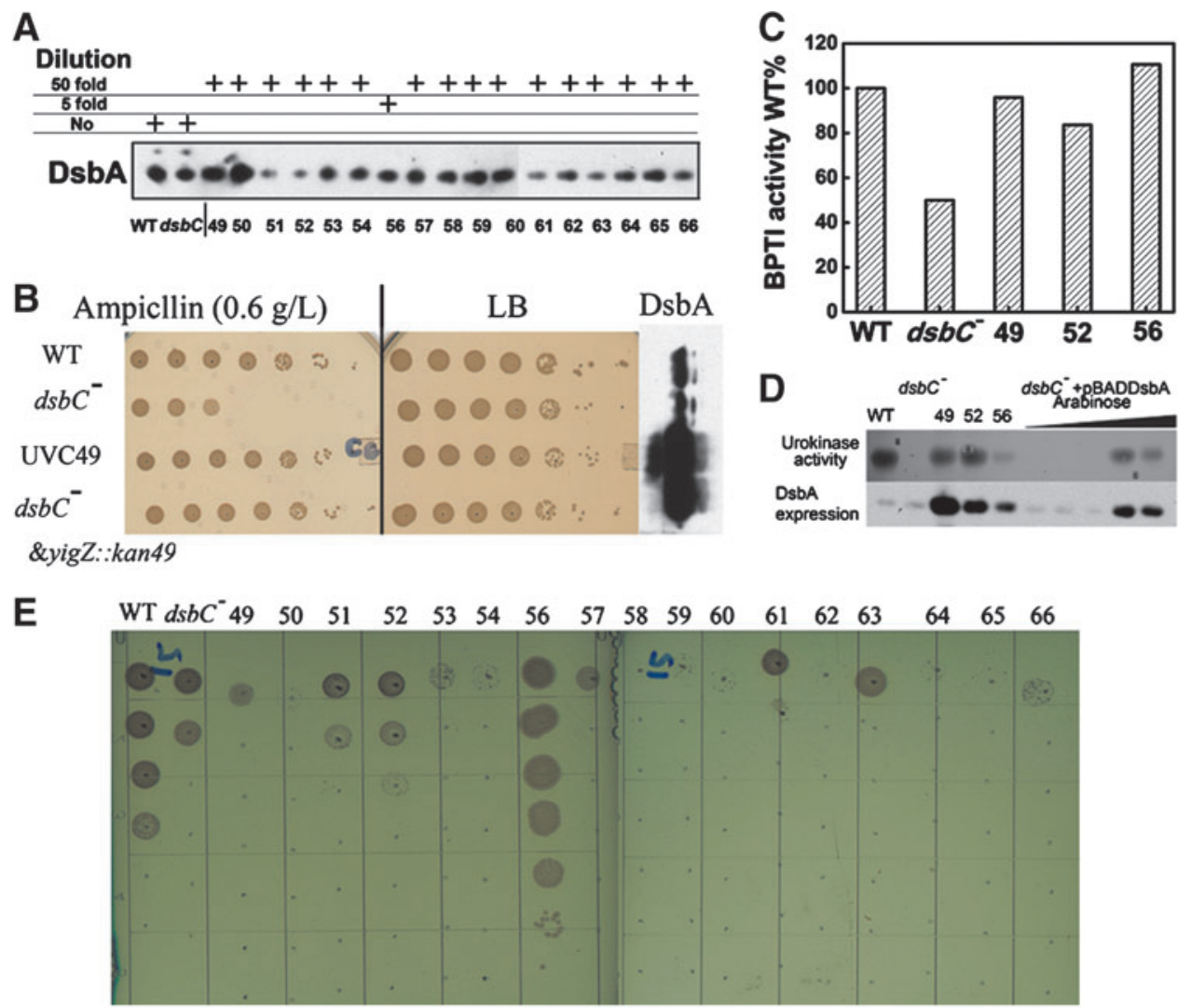

tibody against DsbA is shown at the right. (C) Restoration of bovine pancreatic trypsin inhibitor (BPTI) activity provides an independent indication that the correct disulfide bond formation capacity is restored in these suppressors. BPTI amount was quantified by a trypsin activity inhibition assay. Strains shown are wild type (RGP860), dsbC ${ }^{-}$(RGP862), UVC49 (RGP856), UVC52 (RGP858), and UVC56 (RGP1130). (D) To obtain more evidence that DsbA overexpression in the class I DsbC suppressors is by itself sufficient to rescue the DsbC null phenotypes, we transformed the DsbC null strain with a plasmid that had DsbA expression under the inducible arabinose BAD promotor. Induction of DsbA expression to levels comparable to those present in the class I suppressor UVC52 (by adding arabinose to the media) restored urokinase activity to wild-type levels, confirming that high levels of DsbA overexpression are sufficient to complement $d s b C$. Urokinase activity was detected by a casein agar assay as shown on the upper lane. DsbA expression level was detected by Western blot as shown on the lower lane. Strains shown are wild type (RGP1181), $d s b C^{-}$(RGP1182), UVC49 (RGP1183), UVC52 (RGP1184), UVC56 (RGP1185), and $d s b C^{-}+$pBADDsbA (RGP1244). The triangle indicates increasing amounts of arabinose added to the media at $0 \%, 0.0015 \%$, $0.015 \%, 0.15 \%$, and $1.5 \%$. (E) Copper resistance of $d s b C^{-}$suppressors. UVC56 is more copper resistant than wild-type strains; all other DsbC suppressors were copper sensitive, with most no more copper resistant than the $d s b C^{-}$strain and some even more copper sensitive. Strains used from left to right were RGP663, RGP665, RGP829-834, RGP843-848, RGP852, RGP877-880. (To see this illustration in color the reader is referred to the web version of this article at www.liebertonline.com/ars).

that the presence of cystine or GSSG in minimal media was essential for the oxidase activity of UVC56 (Fig. 7A). That these molecules are ineffective in oxidizing DsbA in wild-type strains appears to be just a function of DsbA expression level because increasing the level of DsbA 5-fold by plasmidmediated expression is sufficient to confer cadmium resistance upon a $d s b B^{-} d s b C^{-}$strain (Fig. 7C). Examination of DsbA's oxidation status by AMS trapping experiments revealed that more reduced DsbA is present in UVC56 than in wild-type strains, both in absolute amount and in the ratio of reduced to oxidized DsbA (Fig. 6B). When the DsbB mutation was replaced by wild-type DsbB, the ratio shifted slightly in the

Table 2. Ampicillin Sensitivity of the yihG::Kan P1 Transduced Suppressor Strains

\begin{tabular}{lcccccccccccccccccccc}
\hline $\operatorname{Amp}(g / L)$ & $W T$ & $d s b C^{-}$ & 49 & 50 & 51 & 52 & 53 & 54 & 56 & 57 & 58 & 59 & 60 & 61 & 62 & 63 & 64 & 65 & 66 \\
\hline 0.2 & 6 & 6 & 6 & 6 & 6 & 6 & 6 & 6 & 6 & 6 & 6 & 3 & 6 & 4 & 6 & 6 & 6 & 6 & 6 \\
1.5 & 6 & 0 & 0 & 0 & 0 & 0 & 0 & 0 & 6 & 0 & 0 & 0 & 0 & 0 & 0 & 0 & 0 & 0 & 0
\end{tabular}

Growth on plates containing $0.2 \mathrm{~g} / \mathrm{L}$ ampicillin was used to indicate the presence of the PDI detector plasmid. Growth of the same strains on plates containing $1.5 \mathrm{~g} / \mathrm{L}$ ampicillin was used to indicate isomerase activity. The values in the $0.2 \mathrm{~g} / \mathrm{L}$ ampicillin row indicate the tested colonies and those in the $1.5 \mathrm{~g} / \mathrm{L}$ row indicate the survivors. Only the wild-type and UVC56 strains remained resistant to $1.5 \mathrm{~g} / \mathrm{L}$ ampicillin after the $d s b A$ region was substituted with wild-type DNA by yihG::kan cotransduction using P1 phage (100\% linkage).

Amp, ampicillin. 
A

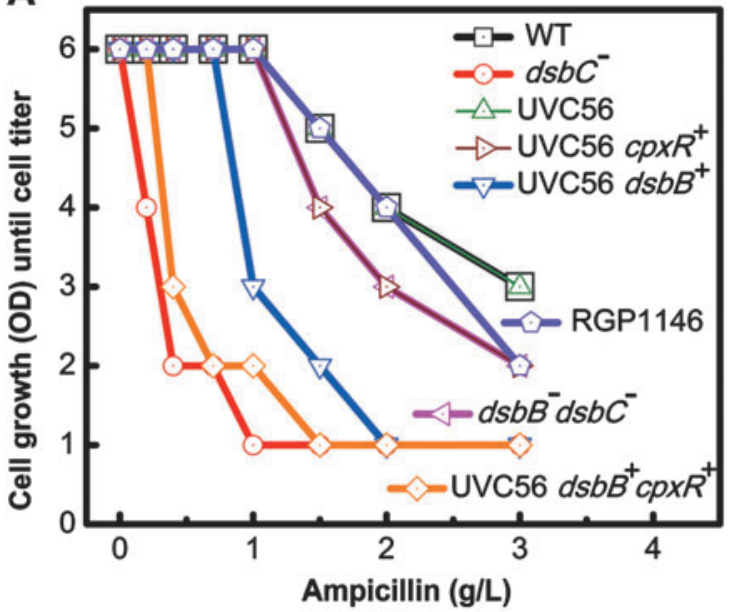

D Temperature

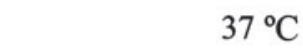

B

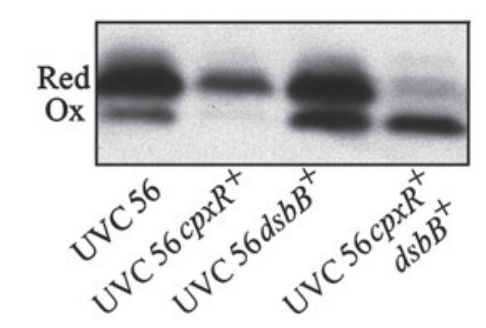

C

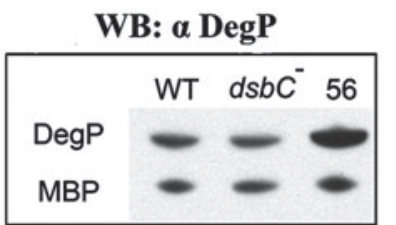

$29^{\circ} \mathrm{C}$

FIG. 6. Mutated $c p x R$ directs DsbA to proofread misoxidized disulfides with the assistance of the chaperone DegP. (A) Ampicillin resistance of UVC56, UVC56 cpx ${ }^{+}, \mathrm{UVC}^{2} 6 d s b B^{+}, \mathrm{UVC} 56$ cpx $R^{+} d s b B^{+}$, and RGP1146 strains; wild-type, $d s b C^{-}$, and $d s b B^{-} d s b C^{-}$strains were used as controls. (B) Analysis of the in vivo oxidation status of DsbA by AMS trapping in UVC56 and wild-type strains (C) Western blot showing upregulation of DegP in UVC56. (D) Copper resistance of UVC56, RGP1146, UVC56 cpxR ${ }^{+}$(RGP1071), UVC56 $d s b A^{-}$(RGP916), and UCV56 $\operatorname{deg} P^{-}$(RGP1256); wild-type and $d s b C^{-}$strains were used as controls. Experiments were done at both $37^{\circ} \mathrm{C}$ where the protease activity of DegP is upregulated and at $29^{\circ} \mathrm{C}$ where DegP's chaperone activity should dominate (38). (To see this illustration in color the reader is referred to the web version of this article at www.liebertonline.com/ars).

oxidized direction. Only when both the $d s b B$ and $c p x R$ genes were wild type was most of the DsbA present in the oxidized form. This result and the fact that DsbA was mostly reduced when only the $c p x R$ gene was made wild type suggest that cpxR also plays a role in determining the redox status of DsbA. CpxR-mediated overproduction of DsbA may make it possible for small molecule disulfides to oxidize DsbA, resulting in a significant portion of DsbA in the oxidized form, though the main fraction remains in the reduced form. Reversion of the $d s b B$ truncation mutation by moving a wild-type $d s b B$ locus into UVC56 resulted in a substantial decrease in ampicillin resistance of UVC56 (compare green and blue traces in Fig. $6 \mathrm{~A})$. Reversion of both the $c p x R$ and $d s b B$ mutations caused the UVR56 strain to be almost as ampicillin sensitive as the starting $d s b C^{-}$strain (compare yellow and red traces in Fig. $6 \mathrm{~A})$. A model that incorporates an important role of both the $c p x R$ and $d s b B$ mutations in the mechanism for class II DsbC suppressors is described in the Discussion.

\section{Discussion}

\section{Mechanism of the class I DsbC suppressors}

Various pathways for the oxidation of substrate proteins by DsbA are shown in Figure 4E. The DsbA-substrate intermediates are shown between steps 1 and $2 \mathrm{a}$, and between steps $2 \mathrm{~b}$ and 3 . Let us first consider the simpler upper reaction pathway consisting of reactions 1 and 2a. In wild-type E. coli, DsbA is maintained mostly in the oxidized form $(\sim>80 \%)$ by the powerful oxidase DsbB; this and DsbA's extremely oxidizing redox potential drive reactions 1 and 2 a to completion. The net effect is that DsbA normally very rapidly oxidizes secreted proteins cotranslationally as they emerge into the periplasm, favoring the oxidation of consecutive cysteine pairs. In our DsbC suppressors the amount of proteins secreted into the periplasm likely remains constant; however, the amount of DsbA clearly increases. A 32-fold higher than normal concentration of oxidized DsbA is present in the periplasm of our class I DsbC suppressors. This was calculated by multiplying the fold overproduction [50] by the fraction oxidized in the suppressor [0.5] by the fraction oxidized in wild type [0.8], that is, $50 \times 0.5 / 0.8$. Given the higher concentration and the strongly oxidizing redox potential of DsbA, it is likely that reaction 1 will be driven forward. The $\sim 125$-fold higher concentration of reduced DsbA present in the periplasm of the suppressors $(50 \times 0.5 / 0.2)$ will tend to increase the rate of the back reaction of step $2 \mathrm{a}$. The percentages of oxidized and reduced DsbA and their absolute amounts as determined by AMS trapping are only approximate, so these calculations are not precise. However, they serve to illustrate the trends. These two effects should tend to increase the concentration of mixed disulfide intermediates, as we observed (Fig. 4D upper panels). Increasing the percentage of substrate proteins that are in the form of mixed disulfides with DsbA increases the opportunities for proteins within this population to fold properly, bringing the correct cysteines into close juxtaposition. Dissociation of DsbA from 
FIG. 7. Characterization of the class II DsbC suppressor UVC56. (A) Cystine or oxidized glutathione (GSSG) in the minimal media fully restores UVC56 motility independent of $d s b B$ but dependent on $d s b A$. Motility assays were performed in minimal media (top) or supplemented with $0.83 \mathrm{mM}$ cystine (bottom left) or $0.83 \mathrm{mM}$ GSSG (bottom right). The strain genotypes are 1, the starting strain $d s b C^{-}$(RGP663); 2, UVC56 (RGP843); 3, UVC56 $d s b A^{-}$(RGP916); 4, UVC56 $d s b B^{-}$(RGP917); 5, UVC56 $d s b D^{-}$(RGP918); 6, UVC56 $d s b G^{-}$(RGP919); and 7, $d s b C^{-} d s b B^{-} \quad$ (RGP433). (B) Cadmium resistance of UVC56, UVC56 cpxR ${ }^{+}$ (RGP1073), and $d s b B^{-} d s b C^{-}$ cpxR56 (RGP1126); wild-type, $d s b C^{-}$, and $d s b B^{-} d s b C^{-}$ strains were used as the controls. Cell titer curves were generated from the gradient spot titer data. (C) Fivefold overexpression of DsbA from plasmid can rescue the cadmium resistance of $d s b B^{-}$

A

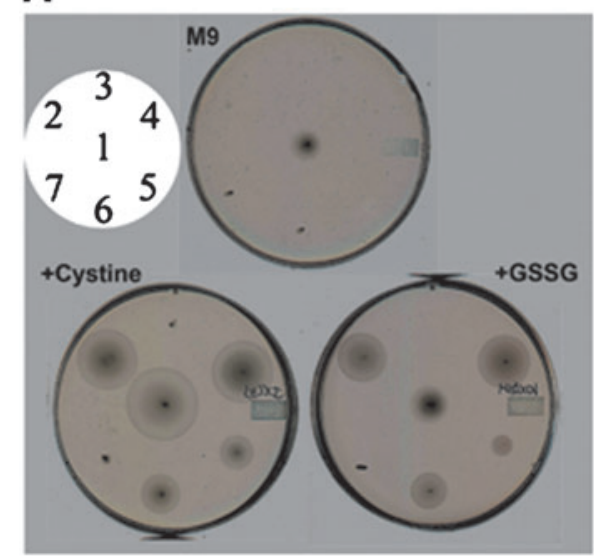

B

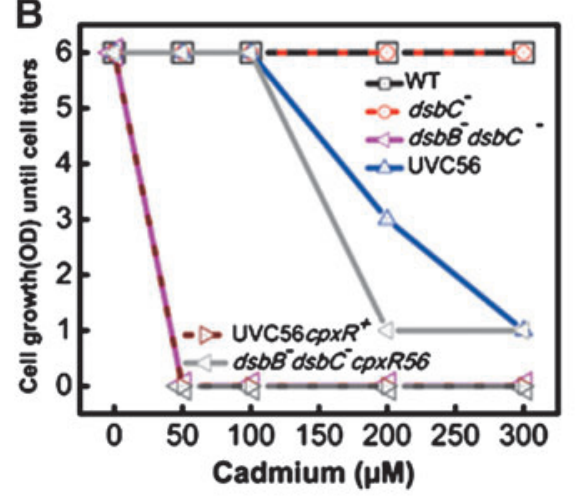

C

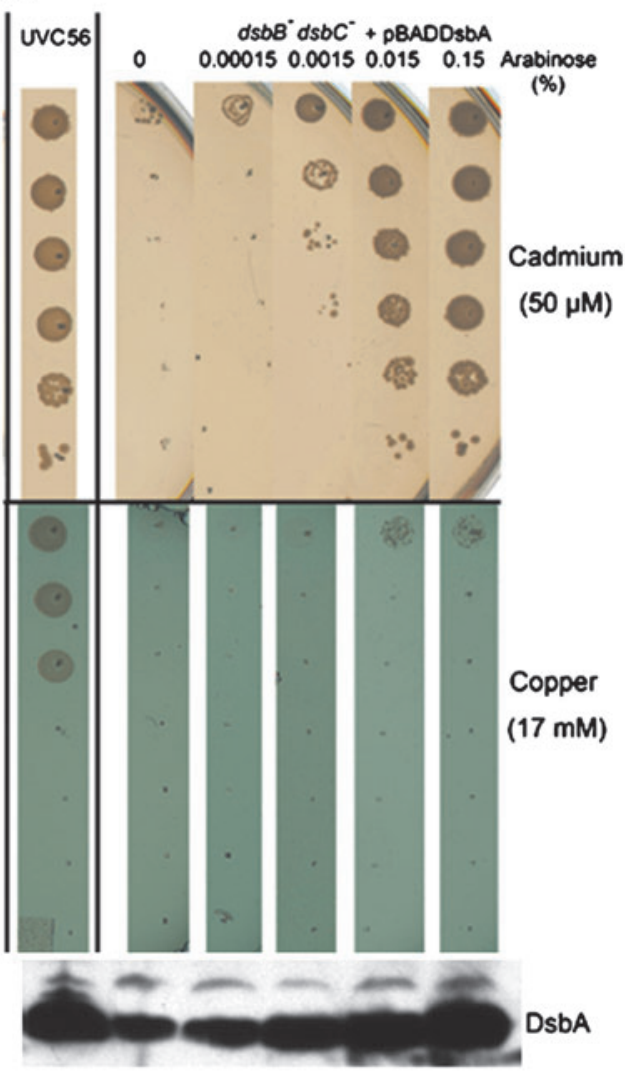
$d s b C^{-}$(RGP1249), but fails to rescue copper resistance. These results suggest that the isomerase activity gained by UVC56 requires more than 5-fold DsbA overexpression. DsbA expression was induced by adding arabinose to the media at the concentrations shown and was confirmed by Western blot of whole cell extracts using antibody against DsbA (bottom panel). (To see this illustration in color the reader is referred to the web version of this article at www.liebertonline.com/ars).

this complex will have the effect of locking in the correct conformation. Because DsbA is a very strong oxidant, reduced DsbA will usually only be effective in reducing incorrect disulfide pairings, which are typically exposed and have relatively high redox potentials. Reduced DsbA will be much less capable of attacking structural disulfides in properly folded proteins, which are typically buried and reducing in redox potential. Thus, once the correct disulfides are formed it will be unlikely that incorrect disulfides will reform.

Another, not mutually exclusive possibility is that the higher level of DsbA leads to a higher level of substrate proteins that, at least transiently, are crosslinked to two or more DsbA molecules via mixed disulfides. The first cysteine on a substrate protein that emerges into the periplasm reacts with DsbA, resulting in the formation of a mixed disulfide (Fig. 4E, step 1). When normal levels of DsbA are present, in the second step (Fig. 4E, step 2a), the second cysteine on the substrate protein to emerge attacks this mixed disulfide. This results in the release of a reduced DsbA molecule and a substrate in which the first two cysteines are joined in a disulfide. Measurements of the rates of these two types of reactions (Fig. 4E, step 1 and step 2a) with GSH and model peptides show that both reactions occur very rapidly (11). A consequence of these rapid rates is that DsbA will very rapidly react with cysteines on secreted proteins as they emerge into the periplasm (25). The rapid rate of resolution of the mixed disulfide dictates that
DsbA will remain in a mixed disulfide with the substrate protein only until an additional cysteine becomes available to resolve the mixed disulfide. Because the periplasm is a very strongly oxidizing environment, free thiols are generally in short supply; the most available source of a nearby free thiol is very likely the next cysteine on the substrate protein that emerges into the periplasmic space. Thus, DsbA tends to be very effective in crosslinking adjacent thiols on proteins, but much less effective in forming nonconsecutive disulfides (6). However, as the concentration of DsbA within the periplasm increases, it will compete more effectively with cysteines that are consecutive in the substrate protein, and instead, mixed disulfides will be formed. As the protein is synthesized, proper folding can occur and the mixed disulfides will be resolved as correct disulfides are formed in a process such as shown in step 3 of Figure 4E. Both of these postulated mechanisms will give the substrate protein better opportunities to fold into its proper conformation, bringing the proper cysteines into proximity and allowing for correct disulfide bond formation.

Copper, a redox active metal, is thought to randomly oxidize unpaired cysteines very rapidly, so that in the presence of copper, it is important to have effective thiol/disulfide isomerases (21). Because DsbC corrects the misoxidized disulfides it confers copper resistance to bacteria (Fig. 6D). Curiously, the $d s b C$ suppressor strains, with the exception of 
UVC56, are not any more resistant to copper than the starting $d s b C^{-}$strain (Fig. 5E). This further suggests the possibility that instead of being more proficient in disulfide isomerization as we had initially predicted, the majority of the $d s b C$ suppressor strains may actually be better at forming the correct disulfides initially, as would be predicted by the reactions shown in Figure 4E. This "do once, do it right" strategy would result in higher levels of urokinase, BPTI, and PDI detector expression but not necessarily increase copper resistance.

\section{Mechanism of class II DsbC suppressors}

We considered a model whereby CpxR-mediated overexpression of $\mathrm{DsbA}$, in conjuction with a shift in the ratio of oxidized toward reduced DsbA due to the DsbB truncation,

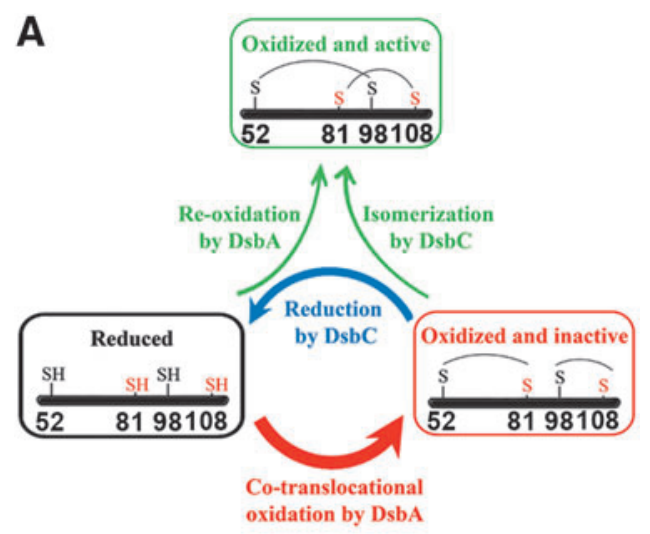

Wild type E. coli

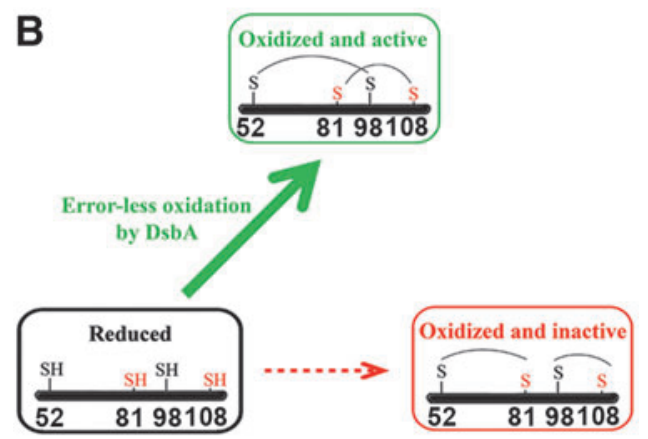

Class I $d s b C^{-}$suppressor

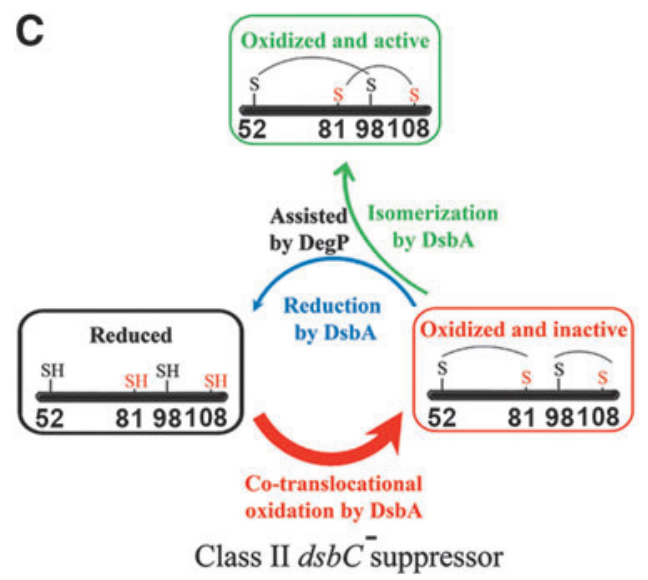

might be sufficient to allow DsbA to effectively function as both an oxidant and as an isomerase in the class II DsbC suppressors. The UVC56 strain is more resistant to copper than is wild-type E. coli, and copper resistance is very closely tied to disulfide isomerization (21). We therefore decided to closely investigate the copper-resistant phenotype of UVC56. DsbA is absolutely necessary for the copper-resistant suppression phenotype (Fig. 6D). Transducing the cpxR56 point mutation into a DsbC null mutation generated a strain that is as copper resistant as UVC56, showing that cpxR56 is also very important for the copper-resistant phenotype. Thus, both the $d s b A$ and $c p x R$ loci are strongly implicated in disulfide isomerization. Our results suggest that maintaining DsbA at the appropriate levels and redox status is important for DsbC suppression in our class II suppressors. However, it is unlikely that simply maintaining DsbA at a proper level and redox status is sufficient for isomerase activity because the class I suppressors showed similar ratios of oxidized to reduced DsbA without gaining copper resistance, and DsbA overexpression from a plasmid is not able to rescue copper resistance (Fig. 7C). In UVC56, the DsbA expression level is not regulated by mutations close to the $d s b A$ locus but by a mutation in $c p x R$, implicating CpxR regulated genes in the isomerase activity. We found through a genetic screen that $d s b A$ and $\operatorname{deg} P$ are both necessary for copper resistance (Fig. 6D). A knockout of DsbA abolished copper resistance, indicating the central role of DsbA in the isomerase activity, and a knockout of DegP strongly decreased copper resistance. A Western blot also showed increased expression of DegP in the UVC56 suppressor strain (Fig. 6C). DegP has been reported to be a protease as well as a

FIG. 8. Potential strategies for forming correct disulfide connectivity patterns in vivo. (A) DsbC-dependent proofreading of substrates with mismatched disulfides. DsbA forms consecutive disulfide bonds cotranslocationally. For substrates that require nonconsecutive disulfides, DsbC either reshuffles them or reduces them followed by another round of DsbA-mediated oxidation. (B) We postulate that the class I suppressor strategy involves DsbA-dependent error-less oxidation of substrates. A high level of DsbA increases the level of DsbA-substrate complexes, which in turn increases the opportunity for correct folding. Correct folding then directly favors correct disulfide bond formation, even in the case where the correct disulfides are nonconsecutive. A small amount of protein as indicated by the red dotted reaction is oxidized consecutively and incorrectly. (C) We postulate that the class II suppressor strategy involves chaperone-assisted DsbA correction of mismatched disulfides. DsbA cotranslocationally oxidizes substrates, forming mixed disulfides with DsbA and preventing formation of incorrect disulfide bonds within the protein. After normal folding processes bring the proper cysteines into proximity, the proper disulfides of the protein are found as shown in Fig 4E. The proteins containing nonnative disulfides are later isomerized by DsbA with the assistance of DegP. The isomerase reactions diagramed in this figure involve a multistep process involving the attack of incorrect disulfides by reduced DsbC or reduced $\mathrm{DsbA}$, which results in the formation of a mixed disulfide between these oxidoreductases and the substrate protein. This is followed by internal rearrangements of the disulfide bonding pattern. This multistep process is simplified in the diagrams by a single arrow labeled isomerization. (To see this illustration in color the reader is referred to the web version of this article at www.liebertonline.com/ars). 
chaperone $(24,29,34,38)$. Our result suggests that DegP can play a role in disulfide isomerization as well. The chaperone activity of DegP may allow it to assist DsbA in disulfide isomerization. Alternatively, the protease activity of DegP might allow it to degrade misoxidized substrates that accumulate to levels exceeding Dsb's isomerization capacity.

\section{Conclusions}

The error-correcting disulfide isomerase activity of DsbC allows it to rearrange the disulfides in those few secreted proteins that require nonconsecutive disulfides (Fig. 8). However, the relatively weak isomerase activity of DsbC may be inadequate to accurately oxidize substrates that contain complicated disulfide patterns. To explore alternate avenues of correct disulfide bond formation, we knocked out the DsbC gene and selected for suppressors that were able to efficiently form nonconsecutive disulfide bonds. The majority of the suppressors we obtained (Fig. 8B, class I) greatly overproduce DsbA and result in a shift toward more reduced DsbA. This enhances the ability of DsbA to facilitate the correct oxidation of proteins, but the copper sensitivity of these suppressors implies that they lack error-fixing ability. We did obtain a suppressor that apparently possesses error-fixing isomerase activity (Fig. 8C, class II suppressor). This suppressor (UVC56) also overproduces DsbA, although to a lesser extent, but in addition, overproduces the chaperone/protease DegP, which apparently enhances the ability of the strain to perform disulfide isomerization reactions.

Eukaryotic proteins often contain complicated disulfide connectivities. For example, tissue plasminogen activator containing 17 disulfides potentially could have over 500 quadrillion disulfide isoforms. This puts extreme demands on cells to accomplish correct disulfide oxidation. Very high levels of DsbA are present in our class I DsbC suppressors, and very high levels of PDI are present in the endoplasmic reticulum of eukaryotes (42). These observations raise the intriguing possibility that PDI may be acting to assist in vivo disulfide formation via a mechanism similar to what we postulate for the DsbA overproducers. That our DsbC suppressors worked in part by shifting the ratio of oxidized to reduced DsbA points out how critical it is to maintain the appropriate thiol disulfide redox balance for enabling proper disulfide bond formation in proteins with multiple cysteines. The emerging role of a redox-driven feedback loop in determining the activity of Ero1 $(1,2,35,36,40)$, the oxidase of PDI, suggests that the redox balance in the endoplasmic reticulum is strictly regulated as well. Elevation of the chaperone/protease DegP in our class II suppressor implies that chaperone activity also plays an important role in disulfide bond isomerization, which is consistent with the previous finding that both PDI and DsbC have chaperone activity $(7,32,44)$. DegP may be able to help fold substrate proteins by bringing nonconsecutive cysteines together in space followed by DsbA functioning as an oxidase. Our ability to select for correct disulfide bond formation in vivo opens up new strategies that may contribute to selecting bacteria with an enhanced capacity to express multidisulfide containing proteins of pharmaceutical importance.

\section{Acknowledgments}

We thank Ursula Jakob and David Ballou for useful suggestions and for critically reading the article. We thank David
Akey for helping to generate Figure 1A. We thank Thomas Silhavy for providing antibody for DegP, and CpxP, and we thank Rajeev Misra for providing antibody against OmpC, OmpA, and OmpF. J.C.A.B. is a Howard Hughes Medical Institute Investigator.

\section{Author Disclosure Statement}

No competing financial interests exist.

\section{References}

1. Appenzeller-Herzog C, Riemer J, Christensen B, Sorensen ES, and Ellgaard L. A novel disulphide switch mechanism in Erolalpha balances ER oxidation in human cells. EMBO J 27: 2977-2987, 2008.

2. Appenzeller-Herzog C, Riemer J, Zito E, Chin KT, Ron D, Spiess M, and Ellgaard L. Disulphide production by Erolalpha-PDI relay is rapid and effectively regulated. EMBO J 29: 3318-3329, 2010.

3. Baba T, Ara T, Hasegawa M, Takai Y, Okumura Y, Baba M, Datsenko KA, Tomita M, Wanner BL, and Mori H. Construction of Escherichia coli K-12 in-frame, single-gene knockout mutants: the Keio collection. Mol Syst Biol 2: 2006.0008, 2006.

4. Bader M, Muse W, Ballou DP, Gassner C, and Bardwell JC. Oxidative protein folding is driven by the electron transport system. Cell 98: 217-227, 1999.

5. Bardwell JC, Lee JO, Jander G, Martin N, Belin D, and Beckwith J. A pathway for disulfide bond formation in vivo. Proc Natl Acad Sci U S A 90: 1038-1042, 1993.

6. Berkmen M, Boyd D, and Beckwith J. The nonconsecutive disulfide bond of Escherichia coli phytase (AppA) renders it dependent on the protein-disulfide isomerase, DsbC. J Biol Chem 280: 11387-11394, 2005.

7. Chen J, Song JL, Zhang S, Wang Y, Cui DF, and Wang CC. Chaperone activity of DsbC. J Biol Chem 274: 19601-19605, 1999.

8. Clarke J and Fersht AR. Engineered disulfide bonds as probes of the folding pathway of barnase: increasing the stability of proteins against the rate of denaturation. Biochemistry 32: 4322-4329, 1993.

9. Combelles C, Gracy J, Heitz A, Craik DJ, and Chiche L. Structure and folding of disulfide-rich miniproteins: insights from molecular dynamics simulations and MM-PBSA free energy calculations. Proteins 73: 87-103, 2008.

10. Danese PN and Silhavy TJ. The sigma(E) and the Cpx signal transduction systems control the synthesis of periplasmic protein-folding enzymes in Escherichia coli. Genes Dev 11: 1183-1193, 1997.

11. Darby NJ and Creighton TE. Catalytic mechanism of DsbA and its comparison with that of protein disulfide isomerase. Biochemistry 34: 3576-3587, 1995.

12. Datsenko KA and Wanner BL. One-step inactivation of chromosomal genes in Escherichia coli K-12 using PCR products. Proc Natl Acad Sci U S A 97: 6640-6645, 2000.

13. Depuydt M, Leonard SE, Vertommen D, Denoncin K, Morsomme P, Wahni K, Messens J, Carroll KS, and Collet JF. A periplasmic reducing system protects single cysteine residues from oxidation. Science 326: 1109-1111, 2009.

14. Depuydt M, Messens J, and Collet JF. How proteins form disulfide bonds. Antioxid Redox Signal 2011 [Epub ahead of print]; DOI: 10.1089/ars.2010.3575.

15. Duguay AR and Silhavy TJ. Quality control in the bacterial periplasm. Biochim Biophys Acta 1694: 121-134, 2004. 
16. Ellgaard L and Ruddock LW. The human protein disulphide isomerase family: substrate interactions and functional properties. EMBO Rep 6: 28-32, 2005.

17. Frech C, Wunderlich M, Glockshuber R, and Schmid FX. Competition between DsbA-mediated oxidation and conformational folding of RTEM1 beta-lactamase. Biochemistry 35: 11386-11395, 1996.

18. Gleiter $S$ and Bardwell JC. Disulfide bond isomerization in prokaryotes. Biochim Biophys Acta 1783: 530-534, 2008.

19. Hansen RE and Winther JR. An introduction to methods for analyzing thiols and disulfides: Reactions, reagents, and practical considerations. Anal Biochem 394: 147-158, 2009.

20. Hatahet $\mathrm{F}$ and Ruddock LW. Protein disulfide isomerase: a critical evaluation of its function in disulfide bond formation. Antioxid Redox Signal 11: 2807-2850, 2009.

21. Hiniker A, Collet JF, and Bardwell JC. Copper stress causes an in vivo requirement for the Escherichia coli disulfide isomerase DsbC. J Biol Chem 280: 33785-33791, 2005.

22. Hiniker A, Ren G, Heras B, Zheng Y, Laurinec S, Jobson RW, Stuckey JA, Martin JL, and Bardwell JC. Laboratory evolution of one disulfide isomerase to resemble another. Proc Natl Acad Sci U S A 104: 11670-11675, 2007.

23. Huang W, Petrosino J, Hirsch M, Shenkin PS, and Palzkill T. Amino acid sequence determinants of beta-lactamase structure and activity. J Mol Biol 258: 688-703, 1996.

24. Jiang J, Zhang X, Chen Y, Wu Y, Zhou ZH, Chang Z, and Sui $\mathrm{SF}$. Activation of DegP chaperone-protease via formation of large cage-like oligomers upon binding to substrate proteins. Proc Natl Acad Sci U S A 105: 11939-11944, 2008.

25. Kadokura $\mathrm{H}$ and Beckwith J. Detecting folding intermediates of a protein as it passes through the bacterial translocation channel. Cell 138: 1164-1173, 2009.

26. Kadokura H, Katzen F, and Beckwith J. Protein disulfide bond formation in prokaryotes. Annu Rev Biochem 72: 111135, 2003.

27. Kadokura H, Tian H, Zander T, Bardwell JC, and Beckwith J. Snapshots of DsbA in action: detection of proteins in the process of oxidative folding. Science 303: 534-537, 2004.

28. Katzen F and Beckwith J. Transmembrane electron transfer by the membrane protein DsbD occurs via a disulfide bond cascade. Cell 103: 769-779, 2000.

29. Krojer T, Sawa J, Schafer E, Saibil HR, Ehrmann M, and Clausen T. Structural basis for the regulated protease and chaperone function of DegP. Nature 453: 885-890, 2008.

30. Messens J, Collet JF, Van Belle K, Brosens E, Loris R, and Wyns L. The oxidase DsbA folds a protein with a nonconsecutive disulfide. J Biol Chem 282: 31302-31307, 2007.

31. Price NL and Raivio TL. Characterization of the Cpx regulon in Escherichia coli strain MC4100. J Bacteriol 191: 1798-1815, 2009.

32. Quan H, Fan G, and Wang CC. Independence of the chaperone activity of protein disulfide isomerase from its thioredoxin-like active site. J Biol Chem 270: 17078-17080, 1995.

33. Rietsch A, Bessette P, Georgiou G, and Beckwith J. Reduction of the periplasmic disulfide bond isomerase, DsbC, occurs by passage of electrons from cytoplasmic thioredoxin. J Bacteriol 179: 6602-6608, 1997.

34. Sawa J, Heuck A, Ehrmann M, and Clausen T. Molecular transformers in the cell: lessons learned from the DegP protease-chaperone. Curr Opin Struct Biol 20: 253-258, 2010.

35. Sevier CS and Kaiser CA. Ero1 and redox homeostasis in the endoplasmic reticulum. Biochim Biophys Acta 1783: 549-556, 2008.
36. Sevier CS, Qu H, Heldman N, Gross E, Fass D, and Kaiser CA. Modulation of cellular disulfide-bond formation and the ER redox environment by feedback regulation of Ero1. Cell 129: 333-344, 2007.

37. Shouldice SR, Cho SH, Boyd D, Heras B, Eser M, Beckwith J, Riggs $\mathrm{P}$, Martin JL, and Berkmen M. In vivo oxidative protein folding can be facilitated by oxidation-reduction cycling. Mol Microbiol 75: 13-28, 2010.

38. Spiess C, Beil A, and Ehrmann M. A temperature-dependent switch from chaperone to protease in a widely conserved heat shock protein. Cell 97: 339-347, 1999.

39. Stafford SJ, Humphreys DP, and Lund PA. Mutations in dsbA and dsbB, but not dsbC, lead to an enhanced sensitivity of Escherichia coli to $\mathrm{Hg} 2+$ and Cd2 +. FEMS Microbiol Lett 174: 179-184, 1999.

40. Tavender TJ and Bulleid NJ. Molecular mechanisms regulating oxidative activity of the Ero1 family in the endoplasmic reticulum. Antioxid Redox Signal 13: 1177-1187, 2010.

41. Vertommen D, Depuydt M, Pan J, Leverrier P, Knoops L, Szikora JP, Messens J, Bardwell JC, and Collet JF. The disulphide isomerase DsbC cooperates with the oxidase DsbA in a DsbD-independent manner. Mol Microbiol 67: 336-349, 2008.

42. Wilkinson B and Gilbert HF. Protein disulfide isomerase. Biochim Biophys Acta 1699: 35-44, 2004.

43. Zapun A and Creighton TE. Effects of DsbA on the disulfide folding of bovine pancreatic trypsin inhibitor and alphalactalbumin. Biochemistry 33: 5202-5211, 1994.

44. Zheng J and Gilbert HF. Discrimination between native and non-native disulfides by protein-disulfide isomerase. J Biol Chem 276: 15747-15752, 2001.

Address correspondence to: Dr. James C.A. Bardwell

Department of Molecular, Cellular, and Developmental Biology Howard Hughes Medical Institute University of Michigan 830 N. University Ave. Ann Arbor, MI 48109-1048

E-mail: jbardwel@umich.edu

Date of first submission to ARS Central, November 16, 2010; date of final revised submission, January 20, 2011; date of acceptance, January 20, 2011.

\section{Abbreviations Used}

AMS $=4$-acetamido-4'-maleimidylstilbene-2,2' disulfonic acid

$\mathrm{BPTI}=$ bovine pancreatic trypsin inhibitor

$\mathrm{DTT}=$ dithiothreitol

$\mathrm{GSH}=$ glutathione

GSSG $=$ oxidized glutathione

$\mathrm{LB}=$ Luria broth

$\mathrm{MBP}=$ maltose binding protein

$\mathrm{PDI}=$ protein disulfide isomerase

$\mathrm{SDS}=$ sodium dodecyl sulfate

tr $x=$ thioredoxin 